\title{
THERMAL FLUCTUATION OF THE MAGNETIC WEIGHT OF AGGLOMERATED MAGNETIC NANOPARTICLES UNDER MANGETIC FIELD: EQUILIBRIUM MODEL
}

\author{
Hackjin KIM \\ Chungnam National University, Taejeon, Korea, hackjin@cnu.ac.kr
}

https://doi.org/10.37904/nanocon.2019.8493

\begin{abstract}
Magnetic nanoparticles experience magnetic force under the magnetic field. The magnetic force can be measured as the magnetic weight of the magnetic nanoparticle sample with a conventional electronic balance. The instantaneous jump of the magnetic weight by the Neel and the Brown mechanisms is observed when the magnetic sample is placed under the magnetic field. The magnetic weight increases slowly thereafter as the nanoparticles agglomerate. During the slow increase of the magnetic weight, the magnetic weight fluctuates with the change of temperature. When the temperature rises, the magnetic weight decreases, and vice versa, which suggests that the magnetic weight could be used as a thermometer. The thermal fluctuation of the magnetic weight results from the structural relaxation of the agglomerate by the kinetic energy of nanoparticles. We propose a simple equilibrium model for the thermal fluctuation of the magnetic weight, which explains the fluctuation in terms of the free energy difference between the agglomerated and the dispersed nanoparticles. The structural relaxation of the agglomerate is a complex process because many different clusters are formed during the agglomeration. In order to understand the details of the thermal fluctuation of the magnetic weight, the energy landscape picture with the distribution of the involved free energy is required.
\end{abstract}

Keywords: Magnetic nanoparticles, magnetic weight, thermal fluctuation, equilibrium model

\section{INTRODUCTION}

Magnetic nanoparticles such as magnetite have been widley used in many different fields such as imaging, medicine, separation, etc. [1] Sometimes the surfaces of magnetite nanoparticles are modified or the nanoparticles are combined with biomolecules for special applications. [2,3] When the magnetic field is applied to magnetic nanoparticles in various applications, they often agglomerate via interparticle interactions, which often hinders the efficiency of the applications. As the magnetic nanoparticles agglomerate under the magnetic field, the magnetization of the sample increases, which means that the nanoparticles experience the greater magnetic force. The magnetic force can be measured as the magnetic weight with a balance. Temporal change of the magnetic weight gives insight on the details of the agglomeration dynamics of magnetic nanoparticles.

In this work, we have built a simple apparatus for the measurement of the magnetic weight. The nonexponential increase of the magnetic weight results from the distribution of the energy barrier involved in the agglomeration. The magnetic weight of the sample fluctuates with the variation of the laboratory temperature. The magnetic weight of the agglomerate decreases with the temperaure rise, and vice versa because the thermal energy of the nanoparticles induces the structural relaxation of the agglomerate. Coherent fluctuation of the magnetic weight with the temperature suggests that the magnetic weight can be used as a temperature sensor. We propose a model for the thermal fluctuation of the magnetic weight, which is based on the equilibrium between the agglomerated and the dispersed nanoparticles. The dispersed superparamagnetic nanoparticles do not contribute to the magnetic weight in the solution.

\section{EXPERIMENTAL}

The experimental setup for monitoring the change of the magnetic weight is shown in Figure 1. [4] The magnetic field is applied with a magnet under the sample and the magnetic weight of the sample is usually 
recorded every minute in the connected computer. The magnetite nanoparticles of $\sim 8 \mathrm{~nm}$ diameter are synthesized following a well-known procedure.[5] In order to study the thermal fluctuation of the magnetic weight, the laboratory temperature is recorded separately. As shown in Figure 2, the magnetite nanoparticles agglomerate in a few hours under the magnetic field, however, the magnetic weight increases steadily for a long time as shown in Figure 3.[4]

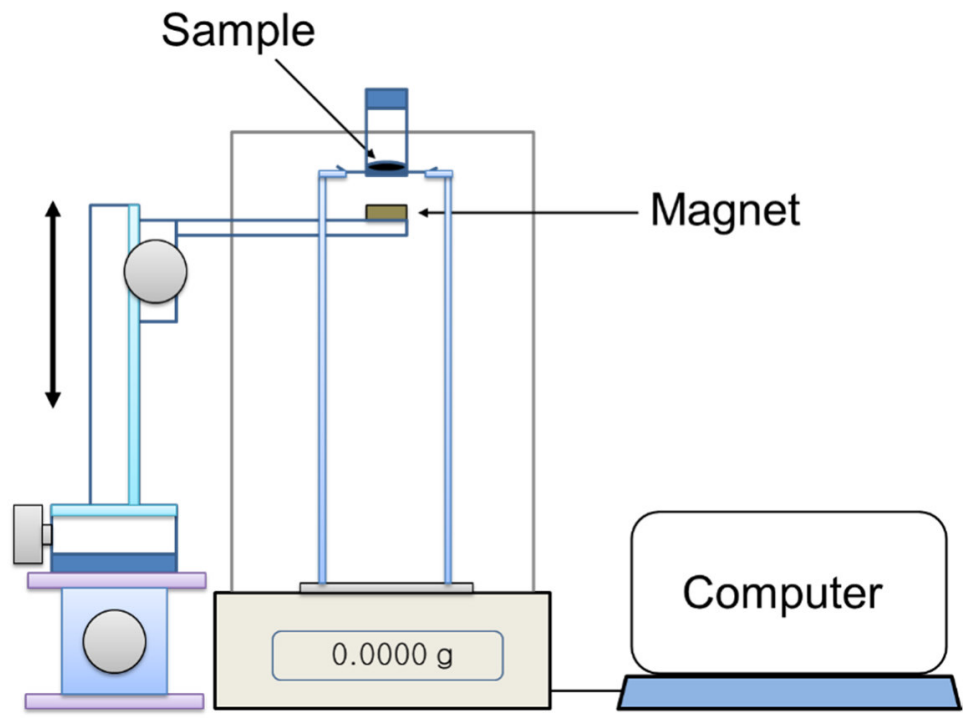

Figure 1 Experimental setup. [4] The conventional electronic balance is used to measure the magnetic weight and the magnetic weight data are stored in the computer connected with the balance. The magnetic field strength is adjusted by moving the magnet under the sample.
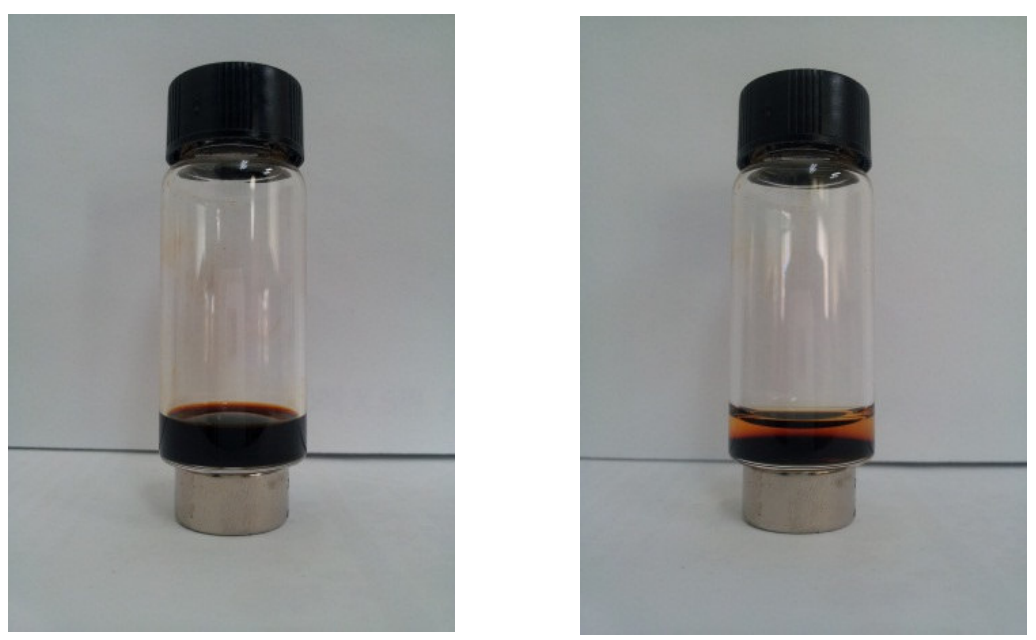

Figure 2 The magnetite nanoparticle sample of $1 \mathrm{wt} \%$ on the magnet. [4] As soon as the sample is placed on the magnet, the surface morphology of the sample is slightly modified as shown in the picture on the left.

The sample solution separates into two phases in a few hours as the nanoparticles agglomerate on the bottom.

\section{RESULTS AND DISCUSSION}

Figure 3 shows the temporal change of the magnetic weight of the samples with different concentrations. [6] The magnetic weight jumps instantaneously by the Neel and the Brown mechanisms and then increases 
slowly. Those relaxation mechanisms occur too fast to monitor the conventional electronic balance. The response time of the balance is $\sim$ a msec and the relaxations occur in the time scale much shorter than $\mu$ sec. The magnetic weight fluctuates around the fitted curve with the variation of the laboratory temperature. The fitted curves of Figure 3(a) are the stretched exponential function. Figure 3(b) shows the ratio of the fitted magnetic weight $\left(\mathrm{W}_{\mathrm{F}}\right)$ and the measured magnetic weight $\left(\mathrm{W}_{\mathrm{M}}\right)$ of Figure $3(\mathrm{a})$. We propose a thermodynamic equilibrium model to explain the fluctuation of the ratio of the magnetic weights.
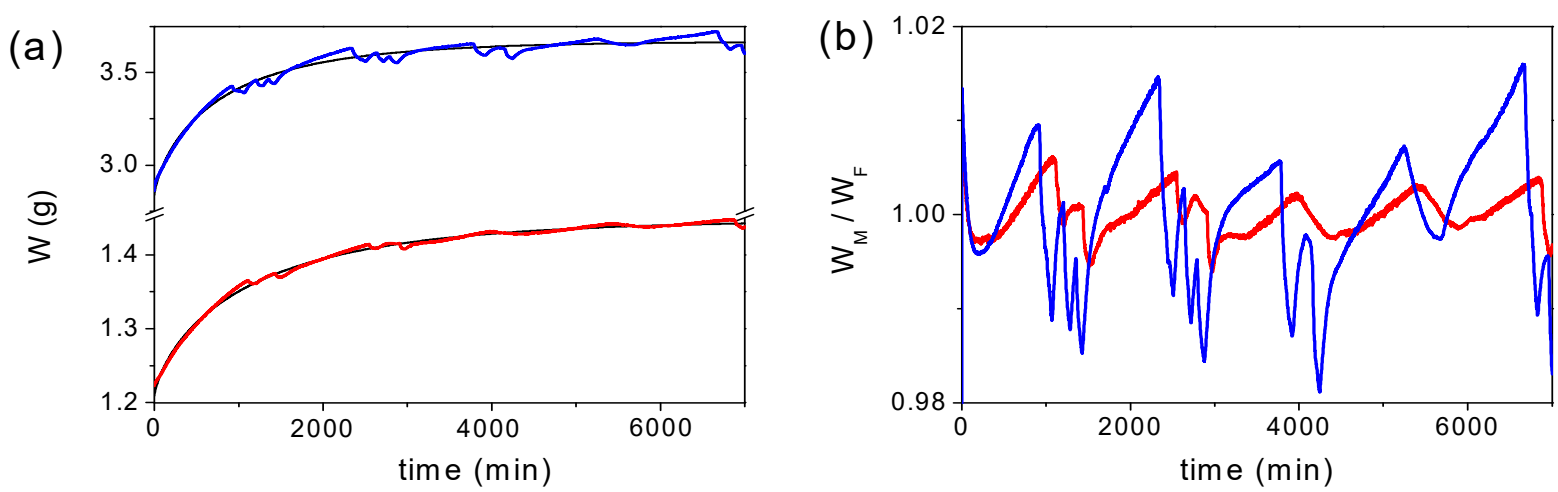

Figure 3 (a) Change of magnetic weight of the samples with different concentrations with the fitted curves [3 wt\%(blue line) and $0.75 \mathrm{wt} \%$ (red line)]. (b) Ratio of the measured $\left(\mathrm{W}_{\mathrm{M}}\right)$ and the fitted $\left(\mathrm{W}_{\mathrm{F}}\right)$ magnetic weight of the $3 \mathrm{wt} \%$ (blue line) and the $0.75 \mathrm{wt} \%$ (red line) sample. [6]

The stretched exponential dependence of a dynamics indicates that the energy barrier involved in the dynamics is not a single value but has a distribution. The stretched exponential function is given by [6]

$\mathrm{W}_{\mathrm{F}}(\mathrm{t})=\mathrm{W}(\infty)+[\mathrm{W}(0)-\mathrm{W}(\infty)] \exp \left(-\left(\frac{\mathrm{t}}{\tau}\right)^{\beta}\right]$

where:

$W(\infty)$ - the final magnetic weight

$\mathrm{W}(0)$ - the initial magnetic weight

$\tau$ - the relaxation time

$\beta$ - the exponent, $0<\beta<1$

The temporal behaviour of the dynamics has a relation with a distribution function of the energy barrier as following [7]

$\mathrm{W}(\mathrm{t})=\int d E g(E) \exp (-k t)$

where:

$g(E)$ - the distribution function of the energy barrier

$k$ - the rate constant

When the rate constant expression such as the Arrhenius type equation and the involved parameters are known, the distribution function of the energy barrier can be determined by the inverse Laplace transform of the temporal behavior of the dynamics. $[7,8]$ 
The therrmal fluctuation of the magnetic weight follows the variation of the laboratory temperature as shown in Figure 4. [6] Those results indicate that the fluctuation of the magnetic weight is not under the kinetic control like the stretched exponential increase of the magnetic weight but under the thermodynamic control.
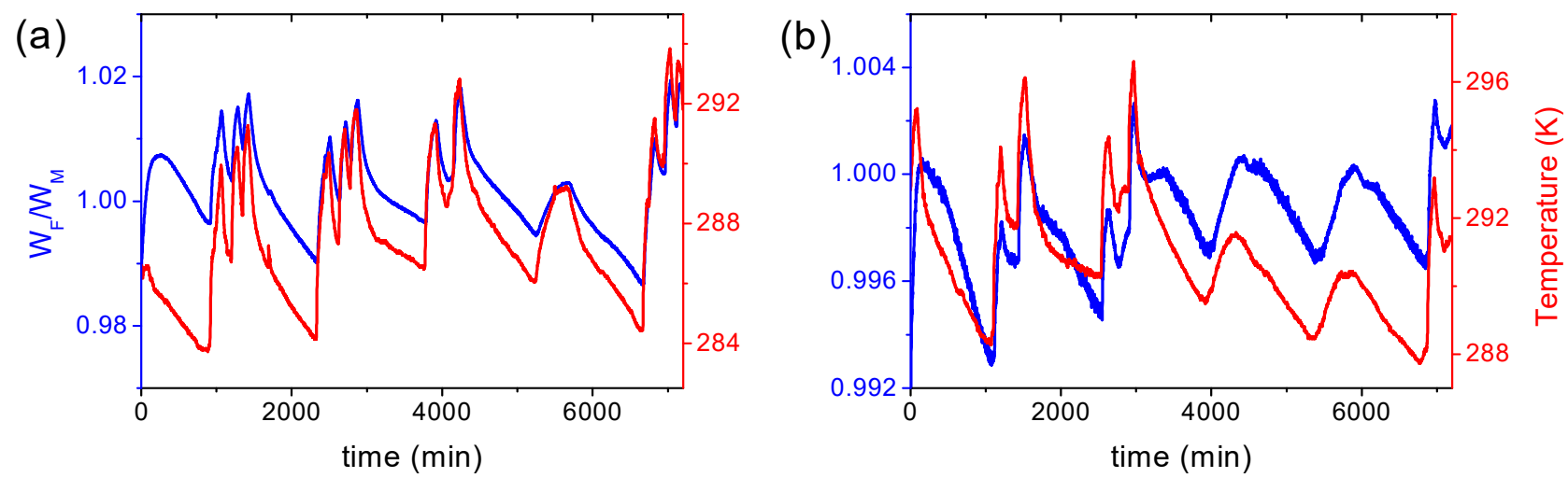

Figure 4 Temporal change of the magnetic weight ratio and the laboratory temperature (a) $3 w t \%$ sample

(b) $0.75 \mathrm{wt} \%$ sample of Figure $3[6]$

\subsection{Equilibrium model [6]}

The temperature dependence of the magnetic weight fluctuation indicates that the fluctuation results from the fast equilibrium of agglomeration. The agglomeration of nanoparticles is the exothermic process, and the entropy of the system decreases as the dispersed nanoparticles agglomerate. Therefore, the equilibrium between the dispersed and the agglomerated state of nanoparticle shifts to the dispersed state with the temperature rise. A single equilibrium constant is not applicable for the agglomeration of nanoparticles if the agglomerate of nanoparticles is composed of many different kinds of nanoparticle clusters.

When the agglomerate is assumed to be composed of clusters or domains with different free energies, the ratio of the measured and the fitted magnetic weight is given by

$\frac{\mathrm{W}_{\mathrm{M}}}{\mathrm{W}_{\mathrm{F}}}=\frac{\left[1+\sum \exp \left(-\frac{\Delta \mathrm{G}_{\mathrm{i}}}{\mathrm{RT}_{\mathrm{a}}}\right)\right]}{\left[1+\sum \exp \left(-\frac{\Delta \mathrm{G}_{\mathrm{i}}}{\mathrm{RT}}\right)\right]}$

where:

$$
\begin{aligned}
& \Delta \mathrm{G} \text { - the free energy } \\
& \mathrm{R} \text { - the gas constant } \\
& \mathrm{T} \text { - the measured temperature } \\
& \mathrm{T}_{\mathrm{a}} \text { - the average temperature }
\end{aligned}
$$

When the free energy is assumed to be distributed evenly and continuously, the summation of equation (3) is replaced with the integration and is converted into the following expression

$\frac{\mathrm{W}_{\mathrm{M}}}{\mathrm{W}_{\mathrm{F}}}=\frac{\left[1+\mathrm{T}_{\mathrm{a}}\right]}{[1+\mathrm{T}]}$

Figure 5 shows the ratio of the magnetic weights with the simulation results using equations (3) and (4). When the nanoparticle concentration is high, complex energy landscape is involved in the agglomeration dynamics. When the concentration is low, the thermal fluctuation is explained with a single equilibrium process. 

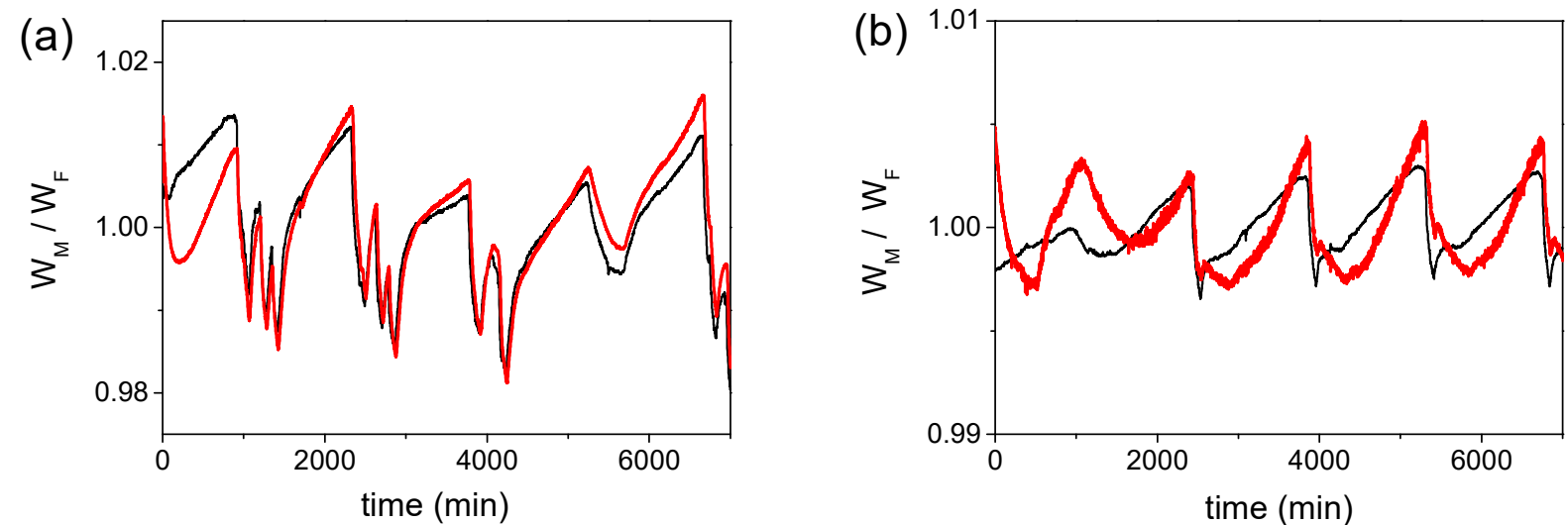

Figure 5 (a) Magnetic weight ratio of the $3 \mathrm{wt} \%$ sample of Figure 3 . The black line corresponds to the ratio simulated with equation (4). (b) Magnetic weight ratio of the $0.75 \mathrm{wt} \%$ sample of Figure 3 . The black line corresponds to the ratio simulated with a single $\Delta G / R(=360)$ of equation (3). [6]

\section{CONCLUSION}

The stretched exponential increase of the magnetic weight of the magnetic nanoparticle sample indicates that the energy barrier of agglomeration has some distribution, that is, the agglomeration process is complex and the energy landscape picture is required in order to understand the complex dynamics of various clusters. Clusters with different sizes and shapes are involved in the agglomeration. The magnetic weight fluctuates with the temperature variation, which suggests that the magnetic weight can be used as a temperature sensor. The thermal fluctuation of the magnetic weight of the agglomerated nanoparticles can be explained with the thermal equilibrium model. Fast equilibrium during the agglomeration is established between the agglomerated and the dispersed nanoparticles.

\section{REFERENCES}

[1] JUN, Y. -W. SEO, J. -W. and CHEON, J. Nanoscaling Laws of Magnetic Nanoparticles and Their Applicabilities in Biomedical Sciences. Accounts of Chemical Research. 2008. vol. 41, no. 2, pp. 179-189.

[2] BARBETA, V. B. JARDIM, R. F. KIYOHARA, P. K. EFFENBERGER, F. B. and ROSSI, L. M. Magnetic properties of $\mathrm{Fe}_{3} \mathrm{O}_{4}$ nanoparticles coated with oleic and dodecanoic acids. Journal of Applied Physics. 2010. vol. 107, no. 7, pp. 073913-1-6.

[3] SARKAR, D. and MANDAL, M. Static and Dynamic Magnetic Characterization of DNA-Templated Chain-Like Magnetite Nanoparticles. Journal of physical chemistry C. 2012, vol. 116, no. 5, pp. 3227-3234.

[4] JIN, D. and KIM, H. Magnetization of Magnetite Ferrofluid Studied by Using a Magnetic Balance. Bulletin of the Korean Chemical Society. 2013. vol. 34, no. 6, pp. 1715-1721.

[5] KANG, Y. RISBUD, S. RABOLT, J. F. and STROEVE, P. Synthesis and Characterization of Nanometer-Size $\mathrm{Fe}_{3} \mathrm{O}_{4}$ and $\gamma-\mathrm{Fe}_{2} \mathrm{O}_{3}$ Particles. Chemistry of Materials. 1996. vol. 8, no. 9, pp. 2209-2211

[6] JIN, D. and KIM, H. Complex Thermal Fluctuation of Agglomerated Magnetic Nanoparticles under Magnetic Field. Bulletin of the Korean Chemical Society. 2019. vol. 40, no. 5, pp. 469-471

[7] AUSTIN, R. H. BEESON, K. EISENSTEIN, L. FRAUENFELDER, H. GUNSALUS, I. C. and MARSHALL V. P. Activation Energy Spectrum of a Biomolecule: Photodissociation of Carbonmonoxy Myoglobin at Low Temperatures. Physical Review Letters. 1974. vol. 32, no. 8, pp. 403-405.

[8] JIN, D. and KIM, H. Stretched Exponential Change of Magnetic Weight of Magnetite Ferrofluid: Distribution of Energy Barrier for Agglomeration of Nanoparticles. Bulletin of the Korean Chemical Society. 2015. vol. 36, no. 1, pp. 424-426 